\title{
Influencia de la testa sobre la imbibición en endocarpios de Malpighia mexicana y Byrsonima crassifolia (Malpighiaceae)
}

\author{
María de los Ángeles Maldonado Peralta', Adelaido Rafael Rojas García', Nicolás Torres Salado', \\ Gabino García de los Santos² , José Rodolfo García Nava² \& Jerónimo Herrera Pérez ${ }^{1}$
}

1. Facultad de Medicina Veterinaria y Zootecnia N²2, Universidad Autónoma de Guerrero, 41940. Cuajinicuilapa, Guerrero, México; mmaldonado@uagro.mx, rogarcia@uagro.mx,nivigas@yahoo.com.mx,mvzjero@hotmail.com

2. PREGEP-Semillas, Botánica, Colegio de Postgraduados, Campus Montecillo. Km. 36.5 Carretera México-Texcoco. 56230, Estado de México: garciag@colpos.mx,garcianr@colpos.mx

Recibido 30-VI-2017 • Corregido 19-IX-2017 • Aceptado 20-X-2017

\begin{abstract}
Influence of testa on endocarp imbibition of Malpighia mexicana and (Byrsonima crassifolia) (Malpighiaceae). Water uptake of seeds is a fundamental internal biochemical activation process. The research was conducted at the Laboratory of Botany and Seeds at the Colegio de Postgraduados, with the objetive of studying the imbibition progress of two species of nanche, subjected to different treatments, in order to asses the water uptake previous to germination process.In the first experiment 80 seeds were placed in petri dishes on moist cotton; in the second, four replications of 100 seeds, each wrapped in a bag and placed in a container with $3 \mathrm{~L}$ of water were taken. Four replications of 25 seeds of each species were used and the head of the embryo was removed, then they were put in petri dishes on wet cotton. The weight was recorded and the rate of imbibition was calculated by subtracting the final weight to the initial, perfoming ANOVA and averages with Tukey $(a=0.05)$. The results showed that Byrsonima crassifolia imbibe low rates and present physical latency, and Malpighia mexicana absorbs twice its weight and loses solutes. The embryo Byrsonima crassifolia absorbs more water while in Malpighia mexicana there is greater imbibition of the seed coat. There were differences between species.
\end{abstract}

Key words: absorption, water, kinetics, embryo, biochemical activation
RESUMEN: La investigación se realizó en el Laboratorio de Semillas y de Botánica del Colegio de Postgraduados, con el objetivo de estudiar la imbibición de endocarpios de dos especies de nanche, sometidos a diferentes tratamientos para determinar la capacidad y velocidad de absorción de agua. En el primer experimento se utilizaron 80 endocarpios de cada especie, colocados en cajas de Petri sobre algodón húmedo; en el segundo, se tomaron 4 repeticiones de 100 endocarpios, cada una, se envolvieron en un saco y se colocaron en un recipiente con 3 $\mathrm{L}$ de agua. En el último, se usaron 4 repeticiones de 25 endocarpios de cada especie, se separó la testa del o los embriones y se colocaron entre cajas de Petri sobre algodón húmedo. El peso se registró y la tasa de imbibición se calculó, realizando ANDEVA y promedios con Tukey $(a=0.05)$. Los resultados muestran que Byrsonima crassifolia imbibe tasas bajas y tiene latencia física, y Malpighia mexicana absorbe el doble de su peso y pierde solutos. El embrión de Byrsonima crassifolia absorbe mayor cantidad de agua mientras que en Malpighia mexicana hay mayor imbibición en la testa. Hubo diferencias entre especies.

Palabras clave: absorción, agua, cinética, embrión, activación bioquímica
El nanche rojo (Malpighia mexicana A. Juss.) y el amariIlo (Byrsonima crasifolia (L.) H. B. K.) son arbustos y árboles con diferentes usos, desde ornamentales, medicinales, artesanales (Jarquín, Martínez, Sánchez, Figueroa, 2011) y nutrimentales, son resistentes a la sequía y a suelos pobres (Fernández \& Rivero, 2004; Herrera y Palomares, 2005). En México se encuentran distribuidos de forma silvestre en zonas tropicales y subtropicales. El nanche amarillo ha comenzado a cultivarse; las plantas silvestres de Malpighia mexicana y Byrsonima crassifolia presentan entre cuatro y cinco picos de producción al año; sin embargo, en muchas especies leñosas se encuentran problemas relacionados con la propagación (Maldonado Peralta et al., 2017), pues en la sexual, aveces la latencia asegura la sobrevivencia en el medio natural, pero dificulta la emergencia (Yépez \& Arboleda, 2009); en nanche la obtención de plántulas por semilla ha sido fundamental para la producción de los cultivos y se indica que presentan bajos índices de germinación (Azerêdo, Matos, Lopes, Da Silva \& Rodrigues, 2005). 
En ambientes de clima seco, el agua se encuentra disponible por periodos cortos, afectando la imbibición de las semillas y por otro lado su germinación (Windauer, Altuna \& Benech-Arnold, 2007). La testa es una barrera que protege a los embriones, permitiendo o no la rápida imbibición de agua (Azcón \& Talon, 2003; Méndez Natera, Merazo, Zerpa \& Bolívar, 2008), donde los bajos porcentajes de germinación comunmente se asocian al endurecimiento de las cubiertas seminales (Zida, Tigabu, Sawadogo \& Oden, 2005), la germinación es lenta y asincronica (Carvalho \& Nascimento, 2008). Además, se ha encontrado que en lotes de semilla de nanche, siempre existen pequeñas proporciones de endocarpios que no presentan dormancia y que germinan en poco tiempo (Carvalho, Nascimento, Müller, 2007).

Las semillas con testa permeable, absorben agua sin importar si son viables o no; de tal forma, que la imbibición depende fuertemente de la permeabilidad de la testa y de la disponibilidad de agua (Méndez et al., 2008). Los tejidos de reserva absorben agua a una velocidad intermedia hasta completar la hidratación (Moreno, Plaza, Magnitskiy, 2006); entre más grande sea la diferencia de contenido de agua en el medio y la semilla, la imbibición será más rápida (Guimarães, Galetti \& Jordano, 2008). Cada especie de nanche es diferente; por ello, cada una requiere de investigación por separado de acuerdo a las características morfológicas que presentan. En campo, el ambiente proporciona condiciones necesarias a las semillas para salir de la latencia (Baskin \& Baskin, 2014); sin embargo, en laboratorio o invernadero, aquellas semillas que presentan lenta germinación, se pueden acelerar mediante tratamientos pre-imbibitorios (Hartmann \& Kester, 1975).

Lopes (1993), Souza (1999) y Souza, Costa, MedeirosFilho y Freitas (1999) reportan que existen especies que no responden a los tratamientos de imbibición, pero Azeredo et al. (2005) indican que la acerola sí responde a éstos tratamientos, pues la absorción de agua a temperatura ambiente durante $72 \mathrm{~h}$ aumentó considerablemente la germinación. Para el caso del nanche amarillo se reporta que los endocarpios a pesar de que presentan testa dura, leñosa y con poca porosidad son permeables al agua, pues al encontrarse disponible la absorben en un periodo máximo de entre 24 y $120 \mathrm{~h}$ (Carvalho \& Nascimento, 2008), al mismo tiempo señalan que la imbibición se lleva a cabo en 3 fases, como indicaron Bewley y Black (1994). Por otra parte estudios realizados en Bauhinia monandra, Thevetia peruviana, Bauhinia ungulata, Enterolobium contortisiliquium y Byrsonima crassifolia (Alves, Medeiros-Filho, Andrade-Neto \& Teófilo, 2000; Cuenca et al., 2003; Contro \& Matos, 2004; Yépez y Arboleda, 2009), usando agua a diferentes temperaturas ha provocado la muerte del embrión y en consecuencia la baja o nula germinación.

Los frutos de nanche rojo tienen tres endocarpios, cada uno con un embrión, proporcional al tamaño del fruto; siendo muy común la ocurrencia de endocarpios vanos, afectando la futura germinación (Maldonado Peralta et al., 2016a). Malpighia punicifolia presenta entre 15 y 30\% de germinación, porcentajes causados por problemas de anormalidades, ineficiencia de agentes polinizadores y otros factores que faltan por estudiarse (Azerêdo, Matos, Germano \& Lima, 1994; Germano, Matos, Azerêdo \& Lima, 1994; Azerêdo et al., 2005). Estudios realizados en endocarpios de acerola (Malpighia glabra L.) han demostrado que en muchos casos existe ausencia del embrión en proporción mayor al 50\% (Musser, Couceiro \& Albuquerque, 1986), lo que se atribuye a posibles problemas de incompatibilidad. Por otra parte, el nanche amarillo tiene un endocarpio con ovario tricarpelado, cada uno para un ovulo, individualizados, pero regularmente uno a dos son viables, rara vez los tres, pero siempre se encuentra la existencia de por lo menos un lóculo, mostrando la fecundación sin haber desarrollo del embrión, además se ha reportado que en esta especie no es recomendable utilizar endocarpios para la propagación, debido a lo complicado que resulta la eliminación de la testa y a los daños causados a los embriones cuando se extraen (Carvalho \& Nascimento, 2008).

En México, el fruto del nanche presenta un bajo índice de comercialización, por lo que no se le ha dado la importancia al valor nutrimental, aceptación del fruto en el mercado y a las condiciones en las que se desarrolla el cultivo; sin embargo, uno de los problemas más serios que presentan es el relacionado a la propagación tanto sexual como asexual; es por ello, que se tiene la necesidad de realizar evaluaciones desde aquellas más simples y que sirvan como base de futuras investigaciones. Por tanto, el objetivo de esta investigación fue estudiar endocarpios de dos especies de nanche (Malpighia mexicana y Byrsonima crassifolia), sometidas a diferentes tratamientos para determinar la influencia de la testa en la imbibición.

\section{MATERIALES Y MÉTODOS}

Para investigar la dinámica de imbibición en el endocarpio, se utilizaron dos especies de nanche, Byrsonima crassifolia y Malpighia mexicana. Los experimentos se ralizaron en el Laboratorio de Análisis de Semillas y de Fisiología Vegetal, Ambiental y Biofísica de Botánica del Colegio de Postgraduados, Campus Montecillo. Los frutos se despulparon friccionandolos en un tamíz metálico 
hasta eliminar la mayor pulpa posible, los endocarpios se enjuagaron con agua y se secaron a la sombra durante 48h, después se realizaron los siguientes experimentos: en el primero se pesaron 20 endocarpios individuales para cada tratamiento; de cada especie, 80 se escarificaron (con una tijera se les eliminó una porción de testa) y 80 se dejaron intactos, utilizandose cuatro repeticiones. Cada repetición se colocó en algodón dentro de cajas de Petri, se humedecieron con igual cantidad de agua destilada $(25 \mathrm{~mL})$, sin inundar, se taparon y se colocaron en una cámara de germinación a $25^{\circ} \mathrm{C}, 90 \%$ de humedad relativa y $12 \mathrm{~h}$ luz, adicionando agua constantemente. Con el uso de una balanza electro-analítica (Scientech ZSA 120), los endocarpios se pesaron cada cuatro horas por doce $h$, posteriormente cada doce $h$ y luego en diferente tiempo, hasta que se obtuvieron pesos constantes o los endocarpios comenzaron a germinar.

En el segundo experimento se utilizaron cuatro repeticiones de 100 endocarpios para cada especie, cada repetición se envolvió en un saco de malla fina de tela, se etiquetaron y pesaron. Se sumergieron en un recipiente con $3 \mathrm{~L}$ de agua destilada a temperatura ambiente, se colocó dentro del recipiente una bomba de aire para oxigenar el agua. Durante $12 \mathrm{~h}$, el peso se determinó cada hora usando una balanza electro-analítica (Scientech ZSA 120), posteriormente, se continuó pesando cada doce $h$ y luego cada veinticuatro $h$ hasta que los pesos se mantuvieron constantes ó disminuyeron. Adicionalmente se realizó un tercer experimento, donde se evaluaron embriones individuales y testa por separado, para ello se contaron cuatro repeticiones de 25 endocarpios de cada especie y con una tijera se abrieron. Sobre cajas de Petri se colocó algodón humedecido con $25 \mathrm{~mL}$ de agua, se colocaron el o los embriones y a un lado la testa, se mantuvieron en una cámara de germinación a $25^{\circ} \mathrm{C}, 90 \%$ de humedad relativa y $12 \mathrm{~h}$ luz, se humedecieron constantemente. El peso se realizó cada h, luego cada doce h, hasta que se observó peso constante o reducción del mismo.

Todos los experimentos se establecieron en un diseño completamente al azar. En el primero, de la combinación realizada resultaron 4 tratamientos (especies $x$ endocarpios escarificados y completos), en el segundo 2 tratamientos (Malpighia mexicana x Byrsonima crassifolia) y el tercero con 2 tratamientos (Malpighia mexicana x Byrsonima crassifolia). La tasa de imbibición se calculó restando al peso de los endocarpios después de la imbibición el peso inicial, se obtuvieron porcentajes, se realizó análisis de varianza y comparación de medias (Tukey $a=0.05$ ) empleando el programa Statiscals Analysis System ${ }^{\circledast}$ (SAS) versión 9.11. Los gráficos se realizaron con el programa SigmaPlot 11.0.Ink.

\section{RESULTADOS}

Primer experimento: La cinética registrada en los endocarpios de las dos especies, mostraron diferencias entre el tiempo y la cantidad de agua imbibibida $(\alpha=0.05)$ (Cuadro 1); donde, en las primeras $4 \mathrm{~h}$ de absorción se alcanzó hasta 21,30 y $74,07 \%$ en semillas completas;

\section{CUADRO 1}

Cinética del tiempo de imbibición de endocarpios completos y escarificados en dos especies de nanche (Byrsonima crassifolia y Malpighia mexicana)

\begin{tabular}{ccccc} 
Especie & \multicolumn{2}{c}{ Byrsonima crassifolia } & \multicolumn{2}{c}{ Malpighia mexicana } \\
Tiempo $(\mathrm{h})$ & Completos & $\begin{array}{c}\text { Escarificados } \\
\text { Contenido de agua de endocarpios (\%) }\end{array}$ & Escarificados \\
\hline 4 & $21,30 \mathrm{~b}$ & $18,63 \mathrm{~b}$ & $74,07 \mathrm{e}$ & $98,59 \mathrm{e}$ \\
8 & $23,58 \mathrm{ab}$ & $20,93 \mathrm{~b}$ & $80,87 \mathrm{e}$ & $110,75 \mathrm{de}$ \\
12 & $24,72 \mathrm{ab}$ & $23,73 \mathrm{ab}$ & $88,63 \mathrm{de}$ & $117,37 \mathrm{cde}$ \\
24 & $26,94 \mathrm{ab}$ & $23,61 \mathrm{ab}$ & $105,72 \mathrm{~cd}$ & $145,96 \mathrm{bcd}$ \\
36 & $28,01 \mathrm{ab}$ & $24,46 \mathrm{ab}$ & $104,72 \mathrm{~cd}$ & $152,66 \mathrm{abcd}$ \\
48 & $29,46 \mathrm{ab}$ & $25,64 \mathrm{ab}$ & $110,81 \mathrm{bc}$ & $159,55 \mathrm{abc}$ \\
60 & $30,69 \mathrm{ab}$ & $28,39 \mathrm{ab}$ & $136,63 \mathrm{a}$ & $180,30 \mathrm{ab}$ \\
120 & $22,93 \mathrm{ab}$ & $28,21 \mathrm{ab}$ & $117,17 \mathrm{bc}$ & $169,27 \mathrm{ab}$ \\
144 & $28,33 \mathrm{ab}$ & $29,99 \mathrm{ab}$ & $125,20 \mathrm{ab}$ & $171,62 \mathrm{ab}$ \\
168 & $31,52 \mathrm{a}$ & $33,50 \mathrm{a}$ & $142,69 \mathrm{a}$ & $191,86 \mathrm{a}$ \\
CV & 24,64 & 29,78 & 11,36 & 18,70 \\
DMS & 10,21 & 11,86 & 19,09 & 43,38 \\
\hline
\end{tabular}

$N=160$ endocarpios por especie. Medias con letras iguales en cada columna, son estadísticamente iguales ( $a=0.05)$. 
mientras que, en los endocarpios escarificados fue de 18,63 y $98,59 \%$, cabe mencionar que son especies diferentes, lo que generó dichos resultados. Los endocarpios de Byrsonima crassifolia son de testa dura y leñosa, considerando éste factor se encontró que estadísticamente no hay diferencias significativas en que imbiban 8 ó 68h; sin embargo, hasta las $60 \mathrm{~h}$ fue donde la absorción fue continua y, posteriormente se encontró disminución de peso hasta las 144h. Los endocarpios escarificados hasta las $12 \mathrm{~h}$ presentaron imbibición continua, luego disminución y aumentos pero sin diferencias estadísticas; por otro lado, en endocarpios completos se alcanzó a observar una fase de la cinética del agua, $y$, en los escarificados dos, incluyendo a la tercera que originó la emergencia de la radícula de la plántula.

Malpighia mexicana presenta endocarpios fibrosos, donde se concentran altas cantidades de agua; los endocarpios completos imbibieron de manera continua durante $24 \mathrm{~h}$, luego se observó una pequeña pérdida que se recupero hasta las $60 \mathrm{~h}$, indicando que es el tiempo adecuado para la imbibición, debido a que lapsos mayores ya no presentan diferencias significativas; la cinética de los endocarpios escarificados mostraron imbibición continua durante $60 \mathrm{~h}$, con 180, $30 \%$ de agua, luego a las $120 \mathrm{~h}$ una pérdida de $11.03 \%$, observandose claramente las fases imbibitorias en ésta especie.

Los endocarpios completos y escarificados de Byrsonima crassifolia imbibieron similar cantidad de agua (Fig. 1); pero a las $4 \mathrm{~h}$ y de las $120 \mathrm{~h}$ en adelante, los no escarificados presentaron entre 2 y $5 \%$ mayor imbibición, con respecto a los escarificados. El experimento se mantuvo hasta las $168 \mathrm{~h}$, tiempo en el que se detuvieron las mediciones debido al inicio de la protrusión de la radícula. Los endocarpios escarificados a las $60 \mathrm{~h}$ iniciaron una reducción de la imbibición, aumentando a las $120 \mathrm{~h}$ pero en menor peso a los no escarificados; en esta especie, la máxima absorción de agua fue de 36,04\%, mientras que en Malpighia mexicana de $188,94 \%$, cantidad equivalente a casi el doble de su peso.

Las dos especies presentaron mayor absorción de agua durante las primeras $4 \mathrm{~h}$ después de establecido el experimento (Fig. 1). Malpighia mexicana presentó tasas relativamente altas en comparación con Byrsonima crassifolia. En Malpighia mexicana se observó que la imbibición fue continua, con picos de mayor absorción a las 24 y $60 \mathrm{~h}$, posteriormente se encontró una reducción, a las 120h inició una recuperación y con mayor aceleración a partir de las 144h. En las dos especies de nanche se presentó diferente cinética del agua; se observó que al inicio imbiben mayor cantidad, luego disminuye para después reiniciarse nuevamente.

Segundo experimento: La cinética del lote de endocarpios sumergidos en el agua mostraron diferencias estadísticas entre el tiempo y la cantidad de agua imbibibida ( $a=0.05$ ) (Cuadro 2); las dos especies alcanzaron la máxima imbibición a las $240 \mathrm{~h}$ pero con descensos intermedios, donde Byrsonima crassifolia presentó una

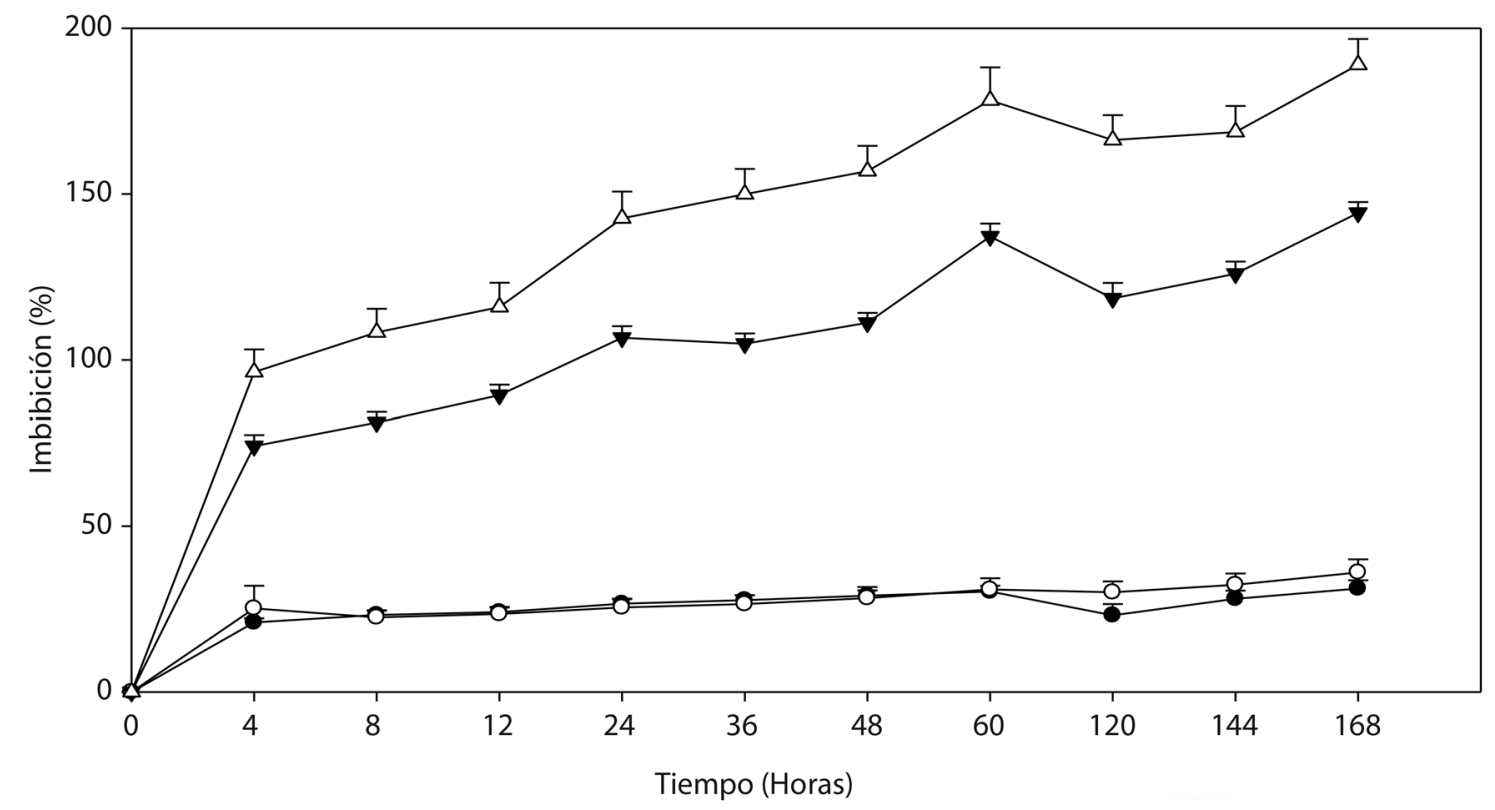

Fig. 1. Dinámica de imbibición de agua en $(\neg-)(-\circ)$ endocarpios completos y $(\neg-)(-\bullet)$ escarificados de Malpighia mexicana A. Juss. y Byrsonima crassifolia (L.) H. B. K., respectivamente. 


\section{CUADRO 2}

Cinética del tiempo de imbibición en lotes de endocarpios completos, en dos especies de nanche (Byrsonima crassifolia y Malpighia mexicana)

\begin{tabular}{ccc}
\hline Especie & $\begin{array}{c}\text { Byrsonima crassifolia } \\
\text { Contenido de agua(\%) }\end{array}$ \\
\hline Tiempo $(\mathrm{h})$ & $39,87 \mathrm{e}$ & $127 \mathrm{e}$ \\
24 & $45,03 \mathrm{~d}$ & $162,97 \mathrm{c}$ \\
48 & $48,13 \mathrm{~d}$ & $149,47 \mathrm{~d}$ \\
72 & $56,65 \mathrm{c}$ & $175,76 \mathrm{~b}$ \\
96 & $55,20 \mathrm{c}$ & $163,02 \mathrm{c}$ \\
120 & $68,09 \mathrm{a}$ & $184,81 \mathrm{a}$ \\
240 & $64,12 \mathrm{~b}$ & $177,96 \mathrm{~b}$ \\
360 & $61,99 \mathrm{~b}$ & $160,65 \mathrm{c}$ \\
480 & 1,36 & 1,36 \\
CV & 3,79 & 3,79 \\
DMS & &
\end{tabular}

$\mathrm{N}=400$ encocarpios por especie. Medias con letras iguales en cada columna, son estadísticamente iguales ( $a=0.05)$.

disminución y Malpighia mexicana dos, siendo a las $48 \mathrm{~h}$ la primera y a las $120 \mathrm{~h}$ la segunda, además de que ésta especie imbibió 3 veces más agua que Byrsonima crassifolia.

A medida que los endocarpios se mantuvieron sumergidos en el agua aumentó la imbibición; sin embargo, éste suceso es hasta cierto tiempo (Fig. 2). El lote de endocarpios de Byrsonima crassifolia presentó 30,59\% de absorción inical, luego fue aumentando lentamente hasta las 96 h, donde hubo una reducción que se recuperó a los 5 días, manteniendose sólo un día y volvió a descender, luego a los 8 días iniciaron pequeños incrementos hasta los 13 días, donde hubo una disminución constante, posteriormente se realizaron observaciones a los embriones y se encontró el 100\% muertos, el remojo prolongado causó esta situación, debido a que la imbibición excesiva provocó muerte por ósmosis de solutos hácia el exterior.

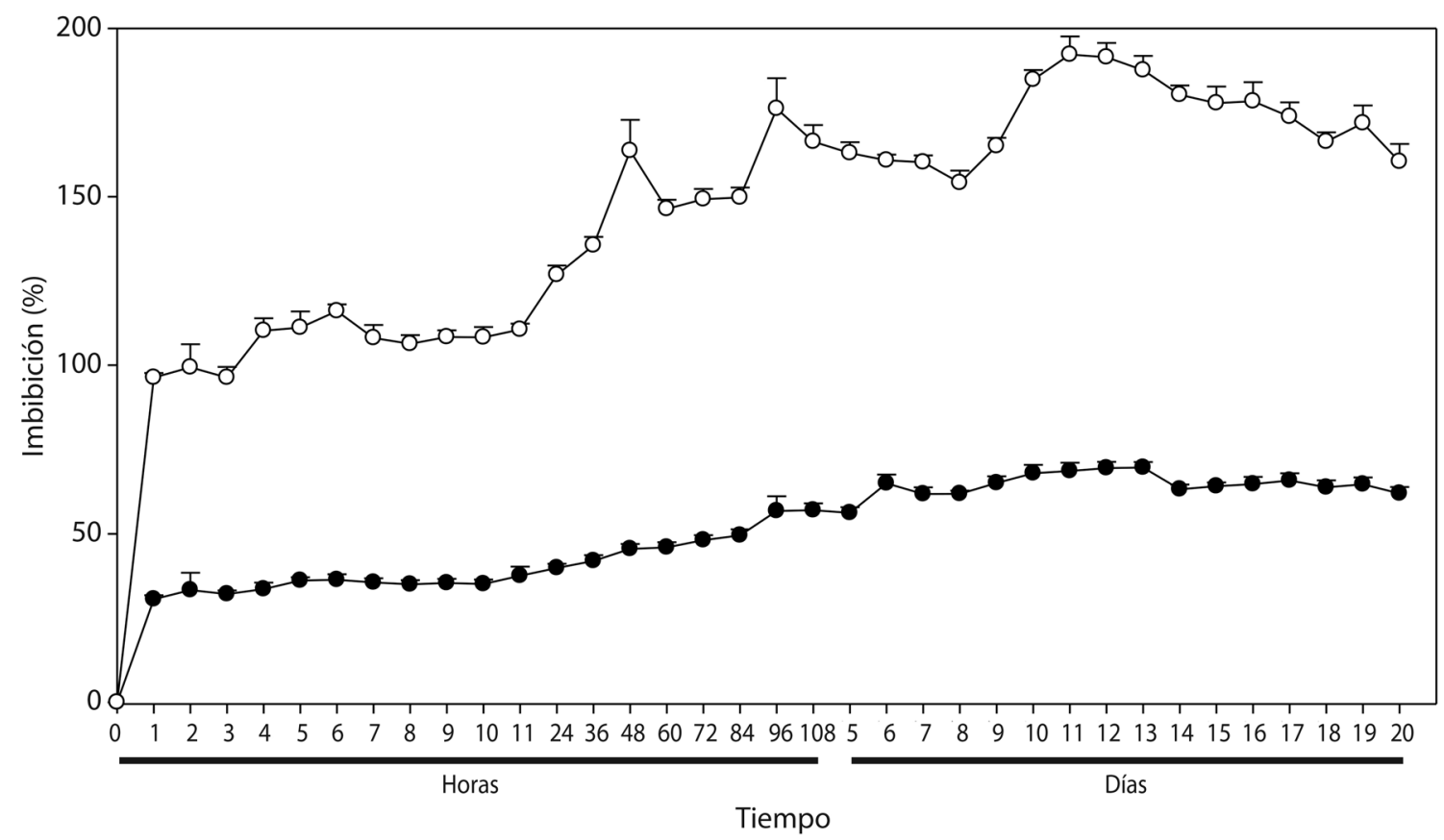

Fig. 2. Dinámica de imbibición, en lotes de 100 endocarpios sin escarificar de $(-\circ)$ Malpighia mexicana A. Juss. y (-) Byrsonima crassifolia (L.) H. B. K. 
El lote de Malpighia mexicana imbibió mayor cantidad de agua (Fig. 2). La respuesta fue similar a la cinética de imbibición realizada en los endocarpios individuales; sin embargo, aquí los endocarpios se mantuvieron en agua durante todo el experimento, ello permitió observar y medir con mayor exactitud. Se encontró que a los $60 \mathrm{~min}$ del establecimiento, Malpighia mexicana imbibió el 96,32\% de agua, posteriormente hasta las $11 \mathrm{~h}$ aumentó su peso pero de forma discreta, continuando con una rápida absorción por $37 \mathrm{~h}$ (hasta las $48 \mathrm{~h}$ ), donde inció un descenso y, a las 60h nuevamente se mantuvo constante, hasta las $84 \mathrm{~h}$, empezando a imbibir nuevamente, pero a las $96 \mathrm{~h}$ descendió hasta los 8 dias, para posteriormente volver a absorber hasta los 11 días, donde comenzó una pérdida sin recuperación.

Tercer experimento: La cinética del agua en embriones y testa de las especies evaluadas registraron diferentes tasas de imbibición en poco tiempo $(\mathrm{a}=0.05)$ (Cuadro 3). El embrión de Byrsonima crassifolia en la primera hora imbibió $85,49 \%$ de agua en relación a su peso, no mostrandose aumentos superiores a éste en el tiempo, pero sí descensos con diferencias estadísticas; además, durante las $53 \mathrm{~h}$ de evalución se encontraron tres fases con aumentos y disminuciones constantes, donde naturalmente había activación interna para el desarrollo del embrión, y la testa o barrera protectora del endocarpio absorbió hasta $39,72 \%$ de agua; Por otro lado, los embriones de Malpighia mexicana imbibieron $69,02 \%$ durante la primera hora de iniciado del experimento, luego disminuyó durante las 2 y $3 \mathrm{~h}$, con recuperación a las $5 \mathrm{~h}$ pero sin diferencias estadísticas, excepto a las $53 \mathrm{~h}$ donde ya hubo mayor disminución y daño del embrión; la testa del endocarpio de ésta especie alcanzó valores de hasta $197,9 \%$, absorbiendo altos porcentajes, como resultado de su morfología.

Los resultados de la Figura 3 muestran que el embrión de Byrsonima crassifolia absorbe agua en mayor cantidad que la testa. Se observó que cuatro horas después de iniciado el experimento, la testa presentó $39,08 \%$ de imbibición; comparando éstos resultados con los de la Figura 1, se observa que un endocarpio completo sin escarificación, en el mismo tiempo absorbe $18,09 \%$ menos que la testa; sin embargo, fue interesante observar que cuando se evaluó el embrión solo, después de la primera hora, absorbió $86,60 \%$ de su peso, después mantuvo un descenso; posteriormente, a las 3 h nuevamente inicia un incremento hasta las $5 h, y$ después se presenta un fuerte descenso de manera constante, situación que no se pudo observar en el experimento 1 y 2 .

Los embriones de Malpighia mexicana presentaron menor imbibición que los de Byrsonima crassifolia (Fig. 3); donde éstos, de Malpighia mexicana una hora después de iniciada la cinética de imbibición, absorbieron $67,13 \%$ de agua, seguido de un descenso continuó hasta las $3 \mathrm{~h}$, posteriormente una recuperación hasta las $5 \mathrm{~h}$ y luego descendieron sin recuperación; durante todo el tiempo de evaluación, en los embriones de las dos especies se encontró una situación semejante. En Malpighia mexicana, la testa imbibió más de $150 \%$, con ascensos y

\section{CUADRO 3}

Cinética del tiempo de imbibición en embrión y testa de endocarpios, en dos especies de nanche (Byrsonima crassifolia y Malpighia mexicana)

\begin{tabular}{ccccc} 
Especie & \multicolumn{2}{c}{ Byrsonima crassifolia } & \multicolumn{2}{c}{ Mallpighia mexicana } \\
Tiempo $(\mathrm{h})$ & Embrión & Testa & Testa \\
\hline 1 & & Contenido de agua (\%) & $157,72 \mathrm{ab}$ \\
2 & $85,49 \mathrm{a}$ & $30,36 \mathrm{a}$ & $69,02 \mathrm{a}$ & $183,53 \mathrm{ab}$ \\
3 & $68,51 \mathrm{abc}$ & $32,51 \mathrm{a}$ & $65,1 \mathrm{a}$ & $145,33 \mathrm{~b}$ \\
4 & $42,21 \mathrm{bc}$ & $31,27 \mathrm{a}$ & $58,24 \mathrm{ab}$ & $180,94 \mathrm{ab}$ \\
5 & $64,65 \mathrm{abc}$ & $39,72 \mathrm{a}$ & $66,49 \mathrm{a}$ & $194,35 \mathrm{a}$ \\
17 & $75,80 \mathrm{ab}$ & $38,75 \mathrm{a}$ & $73,26 \mathrm{a}$ & $181,49 \mathrm{ab}$ \\
29 & $52,63 \mathrm{bc}$ & $36,58 \mathrm{a}$ & $63,99 \mathrm{a}$ & $197,9 \mathrm{a}$ \\
41 & $46,02 \mathrm{bc}$ & $38,29 \mathrm{a}$ & $65,72 \mathrm{a}$ & $170,43 \mathrm{ab}$ \\
53 & $40,54 \mathrm{c}$ & $58,75 \mathrm{ab}$ & $146,5 \mathrm{~b}$ \\
CV & $44,09 \mathrm{bc}$ & $35,83 \mathrm{a}$ & $44,80 \mathrm{~b}$ & 17,86 \\
DMS & 36,85 & 60,16 & 18,51 & 47,09 \\
\hline
\end{tabular}

$N=100$ encocarpios por especie. Medias con letras iguales en cada columna, son estadísticamente iguales $(\alpha=0.05)$. 


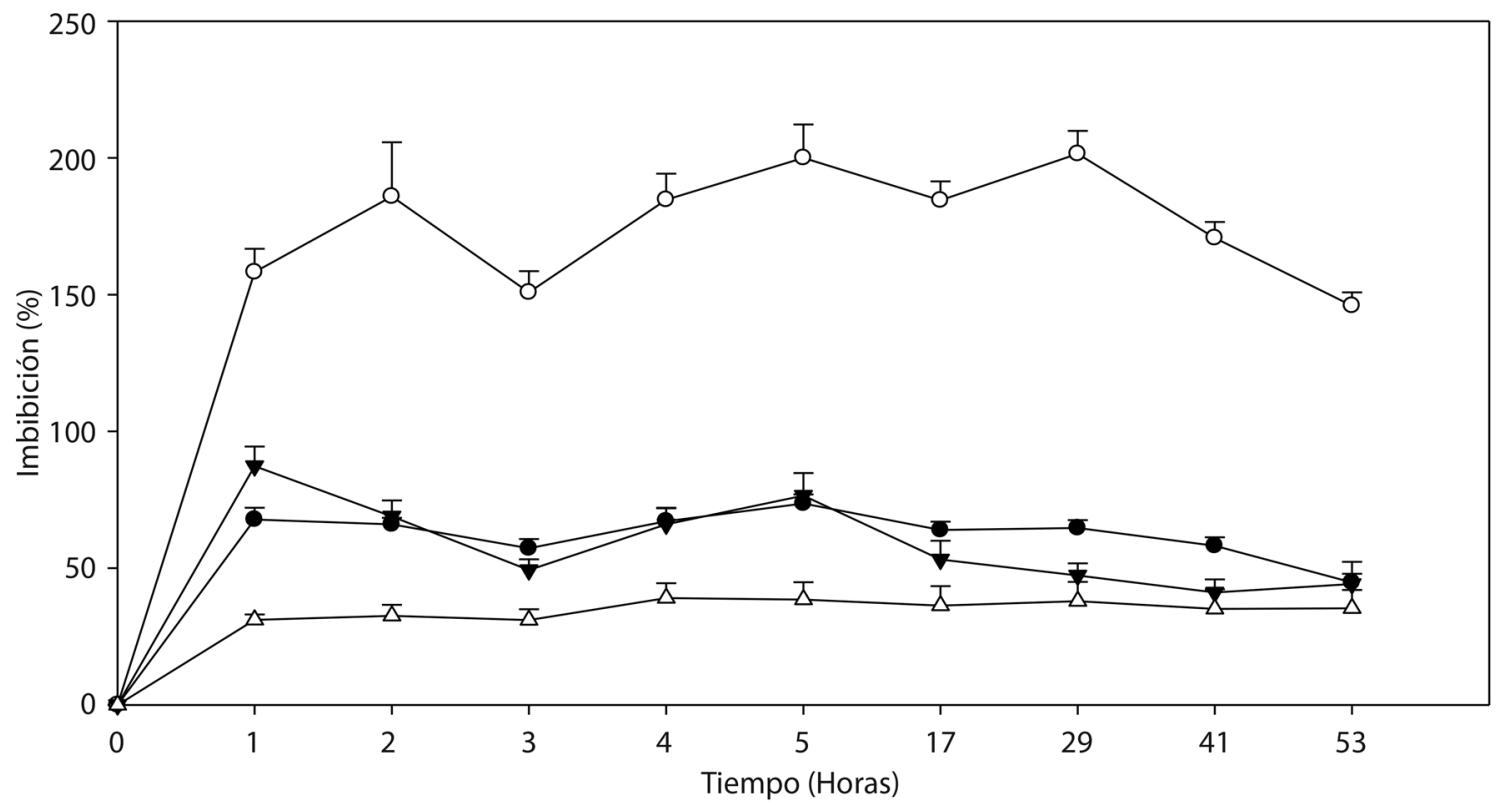

Fig. 3. Dinámica de imbibición en $\left(-0_{-}\right)(-\neg)$ testa y $(-\bullet)(-\bullet)$ embriones por separado, de Malpighia mexicana A. Juss. y Byrsonima crassifolia (L.) H. B. K.).

descensos hasta alcanzar casi $200 \%$, pero a las $29 \mathrm{~h}$ inició un descenso continuó. En Byrsonima crassifolia $4 \mathrm{~h}$ después de iniciada la imbibición, el embrión más la testa absorbieron 105,12 \%, cuando un endocarpio sin escarificar imbibe 20,99\%, encontrandose una diferencia de $126,11 \%$, indicando que los endocarpios de ésta especie presentan baja permeabilidad.

La gráfica comparativa (Fig. 3) indica que la testa de Byrsonima crassifolia no mostró un incremento acelerado inicial del peso, como lo hicieron los embriones, ésta diferencia se debió a la intervención de la testa en el proceso de imbibición, este proceso en las semillas es un factor imprescindible para que se conozca si existe permeabilidad o no; en endocarpios completos de Byrsonima crassifolia, se encontró absorción de apenas un poco más de un tercio de su peso; pero en lotes, ésta misma especie imbibió más de la mitad de su peso. Malpighia mexicana por el tipo de testa fibrosa que presenta, absorbió mayores cantidades de agua que el propio embrion, pues al parecer en esta especie, que desarrolla en zonas tropicales y subtropicales, los embriones en vez de imbibir, liberaron solutos, reduciendo constantemente su peso, la lixiviación de contenidos celulares afectó negativamente los procesos, causando daños negativos. En esta investigación al no conocer a fondo las especies estudiadas se realizó la imbibición del embrión y de su cubierta por separado, encontrando que el embrión tanto de Byrsonima crassifolia y Malpighia mexicana absorbieron rápido y en cantidades cercanas, y la testa imbibió cantidades similares a las del endocarpio completo, interviniendo ésta fuertemente en la entrada de agua hácia el embrión.

\section{DISCUSIÓN}

Cada endocarpio imbibió según el grado de dureza y permeabilidad, dado por la especie; siendo la disponibilidad del agua el principal factor para la cinética de imbibición. Investiaciones realizadas por Azerêdo et al. (2005), mencionan que la imbibición de agua a temperatura ambiente, durante $38 \mathrm{~h}$ en endocarpios de acerola (Malpighia punicifolia) generan altos porcentajes de germinación; caso similar al de ésta investigación, donde el nanche rojo a las $36 \mathrm{~h}$ había imbibido $150 \%$ de agua.

Las dos especies evaluadas respondieron a la imbibición luego de entrar en contacto con el agua, caso que coincide con los estudios realizados en Swietenia macrophylla, donde indican que durante la imbibición hay un incremento considerable en peso (Paiva, Lemos Filho \& Oliveira, 2006), y Méndez Natera, Merazo y Montaño (2008), observaron el mismo patrón de absorción rápida, luego una fase de detención y nuevamente un incremento aunque más lento, terminado por la protrusión de la radícula. Investigaciones realizadas con semillas de maíz indican diferentes fases de aumentos y descensos en las tasas de imbibición, lo que se debe 
a la activación del embrión para su desarrollo y transofrmación a plántula (Rangel Fajardo, Córdoba-Téllez, Cárdenas-Soriano, 2014).

Los endocarpios se mostraron sensibles al exceso de agua, pues la permeabilidad considerada en Byrsonima crassifolia ayuda a que resista y en consecuencia la imbibición se lleve de manera lenta y continua; pero si en ningún momento se elimina el exceso de agua terminan muriendo, lo que indica que los endocarpios, cuando se exponen a humedad en exceso están propensos a sufrir lesiones, caso que también se observó en semillas de soya (Jitsuyana, Hagihara \& Konno, 2014).

Malpighia mexicana es una especie que tiene endocarpios con testa fibrosa, lo que conlleva a que al inicio de la imbibición gran parte del agua sea absorbida por la fribras externas que presenta; sin embargo, las dos especies evaluadas presentaron imbibición, con variación en el tiempo y de acuerdo a la especie en tres ocasiones se encontró aumento y disminución en el peso, de tal manera que en Malpighia mexicana se observaron con mayor exactitud las tres fases de incremento y decremento en la absorción de agua, como lo mencionan Bewley y Black (1994) y Mei y Song (2008); mientras que, en Byrsonima crassifolia al presentar una testa con baja porosidad, provocó que se encontraran incrementos pequeños pero constantes (Carvalho \& Nascimento, 2008).

Byrsonima crassifolia se caracteriza por presentar un endocarpio leñoso con surcos sinuosos y poros pequeños, y en cada uno hay tres embriones ocupando el $64,3 \%$, y sólo el $35,7 \%$ es testa (Maldonado Peralta et al., 2014), la dureza de ésta protege y permite el paso del agua en forma lenta, el embrión sin testa absorbe agua en casi el doble de su peso en poco tiempo, en seguida se observa disminución constante de peso, dando lugar a la activación de eventos internos fisiológicos y bioquímicos (Carvalho \& Nakagawa, 2000).

En endocarpios completos de acerola la imbibición de agua a temperatura ambiente, entre periodos de 38 a 48 h proporcionó mejor viabilidad y vigor durante la germinación (Azerêdo et al., 2005); sin embargo, semillas de Hymenaea courbaril L. respondieron mejor a la germinación cuando la imbibición fue por 24h que a 48h (Orozco-Cardona, Franco-Herrera \& Taborda-Beltrán, 2010). Maldonado Peralta et al. (2016b), investigaron embriones de Malpighia mexicana y Byrsonima crassifolia, y encontraron $90 \%$ de endocarpios viables vigorosos, y que Byrsonima crassifolia presenta latencia física provocada por la testa.

Moreno et al. (2006) mencionan que la testa interviene fuertemente en el proceso de imbibición. Bewley y Black (1994), mencionan que la imbibición es la primera etapa de la germinación y la permeablidad que mostró la testa permitió observar que la absorción de agua es estable durante el proceso y se reduce por la influencia de la testa, esto en gran medida indica que la presencia de la testa en el endocarpio retrasa naturalmente el proceso de germinación y que en condiciones adversas de campo protege la descendencia; también indicaría que los endocarpios de Byrsonima crassifolia presentan latencia física (Maldonado Peralta et al., 2016b), lo que ocasiona que la germinación y el crecimiento de la plántula se retrase (Carvalho \& Nascimento, 2008), y que si no existen condiciones de humedad y temperatura, solo se dañe el endocarpio o el embrión sin que llegue a germinar. Además, cuando se remueve la cubierta el agua entra directo, causando un daño irreparable en el sistema de las membranas embrionales (Castro, Bradford \& Hilhosrt, 2004; Jara, 1996).

Cuando se pretende realizar germinación, es necesario probar la viabilidad de las semillas, después utilizar tratamientos pre-imbibitorios o de escarificación, para ello existen aquellos que cuando se requieren aplicar en gran escala son efectivos (Piroli, Castillo, Vieira \& Udenal, 2005) pero difíciles o lentos (Oliveira, Davide \& Moreira, 2003), lo cual requiere buscar los que sean simples, económicos y que rompan latencia. Tanto para la imbibición como para la germinación, el tiempo se reduce considerablemente cuando se elimina la testa de los endocarpios, seguido por un mayor tiempo al sólo escarificar y aún mayor cuando se dejan éstos intactos (Yépez \& Arboleda, 2009).

La tasa de imbibición para cada especie de nanche es diferente. El endocarpio completo de Byrsonima crassifolia imbibe tasas bajas comparadas con las de Malpighia mexicana, en la que se alcanzó una absorción casi dos veces mayores a su peso. La testa de Malpighia mexicana es la que absorbe mayor cantidad de agua, mientras que en Byrsonima crassifolia es el embrión; sin embargo la testa tiene gran influencia en el proceso de imbibición. Byrsonima crassifolia presentó latencia física, los embriones de las dos especies presentaron comportamiento similar en la imbibición.

\section{REFERENCIAS}

Azcón, B. J., \& Talón, M. (2003). Fundamentos de Fisiología Vegetal. Barcelona, España: McGrawHill/Interamericana.

Alves, A., Medeiros-Filho, S., Andrade-Neto, M., \& Teófilo, E. (2000). Superação da dormência em sementes deBauhinia monandra Brito e Bauhinia ungulata L., Caesalpinoideae. Revista Brasileira de Sementes, 22,139144. doi: 10.17801/0101-3122/rbs.v22n2p139-144 
Azerêdo, G. A., Matos, V. P., Germano, M. L. A. R., \& Lima, A. A. (1994). Efeito da temperatura e períodos de embebição na germinação de sementes de acerola (Malpighia glabra L.). p. 68-69. In Congresso Brasileiro de Fruticultura, 13. Salvador, BA.

Azerêdo, G. A., Matos, P. V., Lopes, P. K., Da Silva, A., \& Rodrigues, de F. L. (2005). Viabilidade e vigor de sementes de acerola (Malpighia punicifolia) submetidas à embebição sob diferentes temperaturas. Pesquisa Agropecuária Tropical, 35,81-84.

Baskin, C. C., \& Baskin, J. M. (2014). Seeds: Ecology, biogeography and evolution of Dormancy and Germination (2da ed.). Kentucky, Estados Unidos: Elsevier. doi: 10.1016/ B978-0-12-416677-6.00006-8

Bewley, J. D., \& Black, M. (1994). Seeds: physiology of development and germination. 2da ed. New York: Plenum. doi: 10.1007/978-1-4899-1002-8

Carvalho, N. M., \& Nakagawa, J. (2000). Sementes: ciência, tecnologia e produção. Jaboticabal, Brasil: FUNEP.

Carvalho, J. E. U. de., Nascimento, W. M. O. do., \& Müller, C. H. (2007). Propagação do Murucizeiro (Byrsonima crassifolia (L.) Rich.). In Carvalho, J. E. U. de; Nascimento, W. M. O. do, \& Müller, C. H. Produção de mudas de espécies frutíferas nativas da Amazônia. Fortaleza, Brasil: Instituto Frutal.

Carvalho, J. E. U. de., \& Nascimento, W. M. O. do. (2008). Caracterização dos pirênios e métodos para acelerar a germinação de sementes de muruci do clone açu. Revista Brasileira de Fruticultura, 30, 775-781. doi: 10.1590/S0100-29452008000300036

Castro, R. D., Bradford, K. J., \& Hilhosrt, H. W. M. (2004). Embebição e reativação do metabolismo. In: Ferreira, A. G., \& Borgheti, F. Germinação: do básico ao aplicado. Porto Alegre, Brasil: Artmed.

Contro, U., \& Matos, M. de. (2004). Dormancy breaking and germination of Enterolobium contortisiliquum (Vell.) Morong seed. Brazilian Archives of Biology and Technology, 47, 851-854. doi: 10.1590/S1516-89132004000600003

Cuenca, G., Zita, D. A., Milagros, I., Laurie, F., Erasmo, M., Milagro, M., \& Rubén, M. (2003). Preselección de plantas nativas y producción de inóculos de hongos micorrízicos arbusculares (HMA) de relevancia en la rehabilitación de áreas degradadas de la gran sabana, Estado Bolívar, Venezuela. Ecotrópicos. Sociedad Venezolana de Ecología, 16, 27-40.

Fernández, C. N., \& Rivero. M. G. (2004). Efecto del ácido indolbútirico (AIB) sobre el enraizamiento de estacas de semeruco (Malpighia glabra L.). Revista Facultad de Agronomía, 21, 42-46.

Germano, M. L. A. R., Matos, V. P., Azerêdo, G. A., \& Lima, A. A. (1994). Influência de diferentes substratos na germinação de sementes de acerola (Malpighia glabra L.). In Congresso Brasileiro de Fruticultura, 13. Salvador, BA.

Guimarães, R. P. Jr., Galetti, M., \& Jordano, P. (2008). Seed Dispersal Anachronisms: Rethinking the Fruits Extinct
Megafauna Ate. Plos ONE, 3, 1-21. doi: 10.1371/journal. pone. 0001745

Hartmann, H. T., \& Kester, E. D. (1975). Plant Propagation: principles and practices. Englewwood Cliffs, Estados Unidos: Prentice-Hall.

Herrera, L. L. E., \& Palomares, V. G. (2005). Escarificación física de semillas de nanche (Byrsonima crassifolia L.) en Xalisco, Nayarit. Tesis profesional para obtener el grado de ingeniero agronómo, Universidad autónoma de Nayarit, México.

Jara, N. L. F. (1996). Biología de las semillas forestales. 2 ed. Turrialba, Costa Rica: CATIE.

Jarquín, L. R., Martínez, M. L., Sánchez, G. J.A., \& Figueroa, J. I. (2011). Parasitoides Asociados a Anthonomus sisyphus Clark (Coleoptera: Curculionidae) en Frutos de Nanche Rojo (Malpighia mexicana) en Oaxaca, México. Southwestern Entomologist, 36, 351-361. doi: 10.3958/059.036.0312

Jitsuyana, Y., Hagihara, Y., \& Konno, Y. (2014). Two imbibition properties independently influence the cultivar-specific flooding tolerance of dried soybean sedes. Seed Science Research, 24, 37-48. doi: 10.1017/S0960258513000378.

Lopes, F. J. S. (1993). Efeito do tempo de embebição em água destilada no vigor de sementes de melão (Cucumis melo L.) Cv. valenciano amarelo. Trabalho de Graduação. Universidade Federal da Paraíba. Terezina, PI.

Maldonado Peralta, M. A., García-Nava, J. R., García De Los Santos, G., Rojas-García, A. R., Cuevas-González, J., \& Torres-Salado, N. (2017). Reguladores del crecimiento y sustratos en la propagación vegetativa de nanche (Malpighia mexicana A. Juss. y Byrsonima crassifolia (L.) H. B. K.). Revista Brasileira de Fruticultura, 39, 1-9. doi: 10.1590/0100-29452017700

Maldonado Peralta, M. A., García De Los Santos, G., García-Nava, J.R., Corona-Torres, T., Cetina-Alcalá, V. M., \& RamírezHerrera, C. (2016a). Calidad morfológica de frutos y endocarpios de nanche rojo (Malpighia mexicana, Malpighiaceae). Acta Botánica Mexicana, 117, 37-46. doi: 10.21829/abm117.2016.1166

Maldonado Peralta, M. A., García De Los Santos, G., GarcíaNava, J.R., Ramírez-Herrera, C., Hernández-Livera, A., Valdéz-Carrazco, J.M., Corona-Torres, T., Cetina-Alcalá, V. M. (2016b). Seed viability and vigour of two nanche species (Malpighia mexicana and Byrsonima crassifolia). Seed Science and Technology, 44, 1-9. doi: 10.15258/ sst.2016.44.1.03

Maldonado Peralta, M. A., García De Los Santos, G., Rojas-García, A. R., García-Nava, J. R., Corona-Torres, T., Cetina-Alcalá, V. M., \& Ramírez-Herrera, C. (2014). Uso de reguladores del crecimiento y sustratos en la propagación vegetativa de nanche (Malpighia mexicana A. Juss.). XXV Congreso Nacional y $V$ Internacional de Fitogenética. 29 de septiembre al 03 de octubre, San Luis Potosí, México.

Mei, Y., \& Song, S. (2008). Early morphological and physiological events occurring during germination of maize seeds. 
Agricultural Sciences in China, 7, 950-957. doi: 10.1016/ S1671-2927(08)60134-0

Méndez Natera, J. R., Merazo, P. J. F., Zerpa, Z. M., \& Bolívar, C. E. (2008). Efecto de la colocación de semillas de maíz (Zea mays L.), caraota (Phaseolus vulgaris L.) y algodón (Gossypium hirsutum L.) en papel toallín (enrollados y sin enrollar) sobre la germinación y el vigor. Revista UDO Agrícola, 8, 67-71.

Méndez Natera, J. R., Merazo, P. J. F., \& Montaño, M. N. J. (2008b). Relación entre la tasa de imbibición y el porcentaje de germinación en semillas de maíz (Zea mays L.), caraota (Phaseoulus vulgaris L.) y quinchoncho (Cajanum cajan (L.) Mill.). Revista UDO Agrícola, 8, 61-66.

Moreno, F., Plaza, G. A., \& Magnitskiy, S. V. (2006). Efecto de la testa sobre la germinación de semillas de caucho (Hevea brasiliensis Muell.). Agronomía Colombiana, 24, 290-295.

Musser, R. S., Couceiro, E. M., \& Albuquerque, M. H. (1986). Efeito do ácido naftaloacético no enraizamento de estacas semi-lenhosas de acerola (Malpighia glabra L.) em sistema de microaspersão. Recife, PE.: UFRPE.

Oliveira, L., Davide, A., \& Moreira, M. (2003). Avaliacao de metodos para quebra da dormencia e para a desinfestacao de sementes de canafistula (Peltophhorum dubium (Sprngel) Taubert. R. Arvore, 27, 597-603. doi: 10.1590/ S0100-67622003000500001

Orozco-Cardona, A. F., Franco-Herrera, N., \& Taborda-Beltrán, L. A. (2010). Evaluación de tres métodos de escarificación en semillas de algarrobo (Hymenaea courbaril L.). Research journal University of Quindio, 20, 36-41.

Paiva, S. E. A., Lemos Filho, J. P., \& Oliveira, D. M. T. (2006). Imbibition of Swietenia macrophylla (Meliaceae) Seeds:
The Role of Stomata. Annals of Botany, 98, 213-217. doi: 10.1093/aob/mcl090

Piroli, E., Castillo, C., Vieira, M., \& Udenal, J. (2005). Germinacao de sementes de canafistula Peltophorum dubium(Spreng) Tamb. tratadas para superacto da dormencia. Colloquium Agrariae, 1, 13-18.

Rangel Fajardo M. A., Córdoba-Téllez, L., \& Cárdenas-Soriano, E. (2014). Pérdida de tolerancia a la desecación durante la imbibición-germinación en semillas de maíz. Revista Mexicana de Ciencias Agrícolas, 5, 833-845.

Souza, F. X. (1999). Préembebição em água e hipoclorito de sódio na germinação de sementes de cajarana (Spondias cytherea Sonn. - Anacardiaceae). Informativo Abrates, 9(1/2), 157.

Souza, F. X., Costa, G. A. M., Medeiros-Filho, S., \& Freitas, S. J. B. (1999). Germinação de sementes de cajazeira (Spondias mombim L. - Anacardiaceae) com pré- embebição em água e hipoclorito de sódio. Informativo Abrates.

Windauer, L. B., Altuna, A., \& Benech-Arnold, R. L. (2007). Hydrotime analysis of Lesquerella fendleri seed germination responses to priming treatments. Industrial Crops and Products, 25, 70-74. doi: 10.1016/j. indcrop.2006.07.004

Yepez, F., \& Arboleda, M. E. (2009). Promoción de la emergencia en urape (Bauhinia monandra Kurz) yretama (Thevetia peruviana (Pers) Schum), especies potenciales para la arboricultura urbana. Bioagro, 21, 15-22.

Zida, D., Tigabu, M., Sawadogo, L., \& Oden, P. (2005). Germination requirements of seeds of four woody species from the Sudanian savanna in Burkina Fasp, West Africa. Seed Science and Technology, 33, 581-593. doi: 10.15258/ sst.2005.33.3.06 